\title{
Thermal model for RHIC, part I: particle ratios and spectra ${ }^{1}$
}

\author{
Wojciech Florkowski and Wojciech Broniowski \\ The H. Niewodniczański Institute of Nuclear Physics \\ ul. Radzikowskiego 152, 31-342 Kraków, Poland
}

\begin{abstract}
A simple thermal model with single freeze-out and flow is used to analyze the ratios of hadron yields and the hadron transverse-mass spectra measured in $\sqrt{s_{N N}}=130 \mathrm{GeV} \mathrm{Au}+\mathrm{Au}$ collisions at RHIC. An overall very good agreement between the model predictions and the data is achieved for all measured hadron species including hyperons.
\end{abstract}

\section{INTRODUCTION}

The main features of the soft hadron production at RHIC, such as the ratios of hadron abundances, the transverse-mass spectra, the elliptic flow, or the HBT radii may be efficiently understood in the framework of a statistical model which combines the standard thermal analysis of the hadron ratios with a suitably parameterized expansion of matter at freeze-out $[1,2,3]$. In the present paper we outline the main assumptions of the model and concentrate on the discussion of the particle ratios $[4,5]$ and the hadron transverse-mass spectra $[6,7,8,9]$. The subsequent paper in these Proceedings [10] contains the analysis of the elliptic flow and the pion HBT correlation radii.

The main ingredients of our approach are as follows: i) the chemical freeze-out and the thermal (kinetic) freeze-out occur simultaneously, ii) all hadronic resonances are included in the calculation of both the hadron yields and the spectra, and iii) the freeze-out hypersurface and the flow at freeze-out are defined by the simple expressions inspired by the Bjorken model [11]. Below we discuss in more detail these three points.

\section{Freeze-out}

Our approach includes a complete set of hadronic resonances in both the calculation of the hadron abundances and the calculation of the hadron spectra. Then, it turns out that the distinction between the two freeze-outs [12] is not necessary. Our analysis showed [4] that the decays of the resonances, which are initially present in a heat bath at the temperature of $165 \mathrm{MeV}$, effectively lower the inverse slope parameters of the spectra by about 30-40 MeV. This is just the desired effect which explains the typical

1 Talk presented at II International Workshop on Hadron Physics, Effective Theories of Low-Energy QCD, 25-29 September 2002, University of Coimbra, Portugal 
difference between $T_{\text {chem }}$ and $T_{\text {kin }}$ (i.e., between the temperature at the chemical freezeout and the thermal freeze-out, respectively). As a consequence, we have found, at least for the RHIC data, that no extra elastic rescattering is required in order to describe simultaneously both the ratios and the spectra. In other words, we assume one universal freeze-out taking place at [1]

$$
T_{\text {chem }}=T_{\text {kin }} \equiv T \text {. }
$$

Recently, experimental hints have been found in favor of our assumption (1). A successful reconstruction of the $K^{*}(892)^{0}$ states by the STAR experiment [13], together with the very good agreement between the measured yield of $K^{*}(892)^{0}$ and the prediction of the thermal model $[3,14]$ suggests a picture with the short expansion time between the two freeze-outs. Such a picture is natural if the production of particles (hadronization) occurs in such conditions that neither elastic or inelastic processes are effective. An example here is provided by the sudden hadronization model of Ref. [15].

\section{Resonances}

For thermal systems the contribution from high-lying (heavy) states is damped by the exponential factor, $\exp \left(-m_{\perp} / T\right)$. This fact, at the first sight, suggests that most of the resonances present in a hadron gas at a moderate temperature may be neglected. However, although the high-lying states are suppressed, their number increases according to the Hagedorn hypothesis $[16,17,18,19]$, such that their net effect turns out to be important. Indeed, hadronic resonances have been included in numerous applications of the statistical models used in the studies of the ratios of hadron multiplicities $[14,20,21,22,23]$ and the effects connected with their decays are essential for the successful description of the data. We note that only a quarter of the observed pions at RHIC comes from the "primordial" pions present at freeze-out, and the remaining three quarters are produced from the decays of resonances.

In our approach, the same number of the resonances is included in the calculation of the hadron ratios and in the calculation of the hadron spectra. In this way, our theoretical spectra have always the correct relative normalization. Moreover, we have worked out semi-analytic formulas for the treatment of the resonance decays [4]. This allows us to sum up exactly many small contributions, especially, those appearing in the sequential decays. All two- and three-body decays are taken into account with the branching ratios taken from the tables. In the case of the three-body decays, the matrix elements are approximated by a constant, hence only the phase-space effect is included.

\section{Expansion}

In order to calculate the spectra we need to specify the expansion of the matter at freeze-out; clearly, the Doppler effect due to flow modifies the spectra and must be properly included. Our choice of the freeze-out hypersurface and the four-velocity at freeze-out has been made in the spirit of Refs. [11, 24, 25, 26, 27, 28, 29, 30] and is defined by the two conditions: 


$$
\tau=\sqrt{t^{2}-r_{x}^{2}-r_{y}^{2}-r_{z}^{2}}=\text { const. }
$$

and

$$
u^{\mu}=\frac{x^{\mu}}{\tau}=\frac{t}{\tau}\left(1, \frac{r_{x}}{t}, \frac{r_{y}}{t}, \frac{r_{z}}{t}\right) .
$$

The constant in Eq. (2) will be later denoted simply by $\tau$. In order to make the transverse size,

$$
\rho=\sqrt{r_{x}^{2}+r_{y}^{2}}
$$

finite, we impose the condition

$$
\rho<\rho_{\max } .
$$

We note that the four-velocity (3) defining the hydrodynamic flow at freeze-out is proportional to the coordinate (Hubble-like expansion). This form of the flow and the fact the the coordinates $t$ and $r_{z}$ are not limited and appear in the boost-invariant combination in (2) mean that our model is boost-invariant. In practical calculations it is convenient to introduce the following parameterization [28]:

$$
\begin{aligned}
t & =\tau \cosh \alpha_{\|} \cosh \alpha_{\perp}, \quad r_{z}=\tau \sinh \alpha_{\|} \cosh \alpha_{\perp}, \\
r_{x} & =\tau \sinh \alpha_{\perp} \cos \phi, \quad r_{y}=\tau \sinh \alpha_{\perp} \sin \phi
\end{aligned}
$$

where $\alpha_{\|}$is the rapidity of the fluid element, $v_{z}=r_{z} / t=\tanh \alpha_{\|}$, and $\alpha_{\perp}$ describes the transverse size, $\rho=\tau \sinh \alpha_{\perp}$. The transverse velocity is $v_{\rho}=\tanh \alpha_{\perp} / \cosh \alpha_{\|}$. The element of the hypersurface is defined as

$$
d \Sigma_{\mu}=\varepsilon_{\mu \alpha \beta \gamma} \frac{\partial x^{\alpha}}{\partial \alpha_{\|}} \frac{\partial x^{\beta}}{\partial \alpha_{\perp}} \frac{\partial x^{\gamma}}{\partial \phi} d \alpha_{\|} d \alpha_{\perp} d \phi
$$

where $x^{0}=t, x^{1}=r_{x}, x^{2}=r_{y}, x^{3}=r_{z}$, and $\varepsilon_{\mu \alpha \beta \gamma}$ is the Levi-Civita tensor. A straightforward calculation yields

$$
d \Sigma^{\mu}(x)=u^{\mu}(x) \tau^{3} \sinh \left(\alpha_{\perp}\right) \cosh \left(\alpha_{\perp}\right) d \alpha_{\perp} d \alpha_{\|} d \phi .
$$

Equation (8) shows that the four-vectors $d \Sigma^{\mu}$ and $u^{\mu}$ are parallel. In this case the spectra may be obtained from the expression analogous to the Cooper-Frye [31,32] formula

$$
\frac{d N}{d^{2} p_{\perp} d y}=\int p^{\mu} d \Sigma_{\mu} f(p \cdot u)
$$

but with the distribution $f$ which has collected the products of resonance decays (for details see [3]). With parameterization (6) we can rewrite Eq. (9) in the form

$$
\frac{d N}{2 \pi m_{\perp} d m_{\perp} d y}=\tau^{3} \int_{-\infty}^{+\infty} d \alpha_{\|} \int_{0}^{\rho_{\max } / \tau} \sinh \alpha_{\perp} d\left(\sinh \alpha_{\perp}\right) \int_{0}^{2 \pi} d \xi p \cdot u f(p \cdot u)
$$


where

$$
p \cdot u=m_{\perp} \cosh \alpha_{\|} \cosh \alpha_{\perp}-p_{\perp} \cos \xi \sinh \alpha_{\perp} .
$$

One can notice that the spectrum (10) is, as expected from the assumed boost invariance, independent of the rapidity $y$.

\section{RATIOS OF HADRON ABUNDANCES}

In the case of the boost-invariant systems, the ratios of hadron multiplicities at midrapidity, $d N /\left.d y\right|_{y=0}$, are simply related to the ratios of the local densities, $n_{i}$, since

$$
\left.\frac{d N_{i} / d y}{d N_{j} / d y}\right|_{y=0}=\frac{N_{i}}{N_{j}}=\frac{n_{i}}{n_{j}}
$$

The first part of this equality follows from the boost invariance, whereas the second part is a consequence of the factorization of the volume of the system (this point is discussed in detail in Ref. [10]). Since the firecylinder formed at RHIC is approximately boost-invariant (at least within one unit of rapidity at $y=0$, which is sufficient for our considerations which concentrate only on the central region), the ratios at zero rapidity measured for various particles may be used to fit the thermal parameters of the model. This is an important observation indicating that the ratios are not sensitive to the particular form of expansion. Consequently, the parameters of our model can be fixed in two steps: with help of the ratios we first fix the thermodynamic parameters, and later with help of the spectra we fix the two expansion parameters, $\tau$ and $\rho_{\max }$.

The density of the $i$ th hadron species is calculated from the ideal-gas expression

$$
\begin{aligned}
n_{i} & =g_{i} \int d^{3} p f^{(i)}(p), \\
f^{(i)}(p) & =\frac{1}{(2 \pi)^{3}}\left(\exp \left[\left(E_{i}(p)-\mu_{B} B_{i}-\mu_{S} S_{i}-\mu_{I} I_{i}\right) / T\right] \pm 1\right)^{-1}
\end{aligned}
$$

where $g_{i}$ is the spin degeneracy, $B_{i}, S_{i}$, and $I_{i}$ denote the baryon number, strangeness,

and the third component of isospin, and $E_{i}(p)=\sqrt{p^{2}+m_{i}^{2}}$. The quantities $\mu_{B}, \mu_{S}$ and $\mu_{I}$ are the chemical potentials which enforce the corresponding conservation laws. We recall that Eq. (13) is used to calculate the "primordial" densities of stable hadrons as well as of all resonances at freeze-out, which decay later on.

Initially, the temperature, $T$, and the baryon chemical potential, $\mu_{B}$, were fitted with the $\chi^{2}$ method to the 9 preliminary experimental ratios of the hadron yields (for the list of the ratios used in this fit and for more details of our analysis see Refs. [4, 5]). The $\mu_{S}$ and $\mu_{I}$ were determined with the conditions that the initial strangeness of the system is zero, and the ratio of the baryon number to the electric charge is the same as in the colliding nuclei. This procedure gave us $T=165 \pm 7 \mathrm{MeV}$ and $\mu_{B}=41 \pm 5 \mathrm{MeV}$. In Table I we present the result of the fit with a wider set of the up-to-date available hadronic ratios. It yields $T=168 \pm 5 \mathrm{MeV}$ and $\mu_{B}=41 \pm 4 \mathrm{MeV}$. It is interesting to 
TABLE 1. Optimal thermal parameters and the ratios of hadron multiplicities at zero rapidity.

\begin{tabular}{cc} 
Model & Experiment \\
\hline
\end{tabular}

Fitted thermal parameters

$\begin{array}{lr}T[\mathrm{MeV}] & 168 \pm 5 \\ \mu_{B}[\mathrm{MeV}] & 41 \pm 4 \\ \mu_{S}[\mathrm{MeV}] & 10 \\ \mu_{I}[\mathrm{MeV}] & -1 \\ \chi^{2} / n & 0.6\end{array}$

Theoretical ratios and the data

\begin{tabular}{|c|c|c|}
\hline $\begin{array}{l}\pi^{-} / \pi^{+} \\
\bar{n} / \pi^{-}\end{array}$ & $\begin{array}{l}1.02 \\
0.09\end{array}$ & $\begin{array}{c}1.00 \pm 0.02[35], \quad 0.99 \pm 0.02[36] \\
0.08 \pm 0.01[37]\end{array}$ \\
\hline & & $0.92 \pm 0.03[38], \quad 0.93 \pm 0.07[39]$ \\
\hline$K^{-} / K^{+}$ & 0.92 & $0.91 \pm 0.09[35], \quad 0.92 \pm 0.06[36]$ \\
\hline$K^{-} / \pi^{-}$ & 0.16 & $0.15 \pm 0.02[40]$ \\
\hline$K_{0}^{*} / h^{-}$ & 0.047 & $0.042 \pm 0.011[13]$ \\
\hline$\overline{K_{0}^{*}} / h^{-}$ & 0.042 & $0.039 \pm 0.011[13]$ \\
\hline $\bar{p} / p$ & 0.65 & $\begin{array}{ll}0.61 \pm 0.07[37], & 0.64 \pm 0.08[39] \\
0.60 \pm 0.07[35], & 0.61 \pm 0.06[36]\end{array}$ \\
\hline $\bar{\Lambda} / \Lambda$ & 0.69 & $0.71 \pm 0.04[38]$ \\
\hline $\bar{\Xi} / \Xi$ & 0.77 & $0.83 \pm 0.06[38]$ \\
\hline$\phi / h^{-}$ & 0.020 & $0.021 \pm 0.001[41]$ \\
\hline$\phi / K^{-}$ & 0.15 & $0.13 \pm 0.03[41]$ \\
\hline$\Lambda / p$ & 0.47 & $0.49 \pm 0.03[42,43]$ \\
\hline$\Omega^{-} / h^{-}$ & 0.0011 & $0.0012 \pm 0.005[44]$ \\
\hline$\Xi^{-} / \pi^{-}$ & 0.0072 & $0.0088 \pm 0.0020[45]$ \\
\hline$\Omega^{+} / \Omega^{-}$ & 0.86 & $0.95 \pm 0.16[38]$ \\
\hline
\end{tabular}

observe that the newly released data are fully consistent with the thermal picture and only a small change of the thermodynamic parameters follows when the new set of the ratios is used.

A characteristic feature of our fit is that the optimal temperature is consistent with the value for the deconfinement phase transition obtained from the QCD lattice simulations: $T_{c}=154 \pm 8 \mathrm{MeV}$ for three massless flavors and $T_{c}=173 \pm 8 \mathrm{MeV}$ for two massless flavors [33]). This type of the behavior has been also found in other statistical calculations $[14,34]$ and is interpreted as the argument for a scenario in which the hadronic ratios are fixed just in the hadronization process.

\section{TRANSVERSE-MASS SPECTRA}

Having fixed the two independent thermodynamic parameters of the model, we can use Eq. (10) to fit the transverse-mass spectra and to fix the two remaining geometric parameters of the model. This method has been initially applied in the study of the 


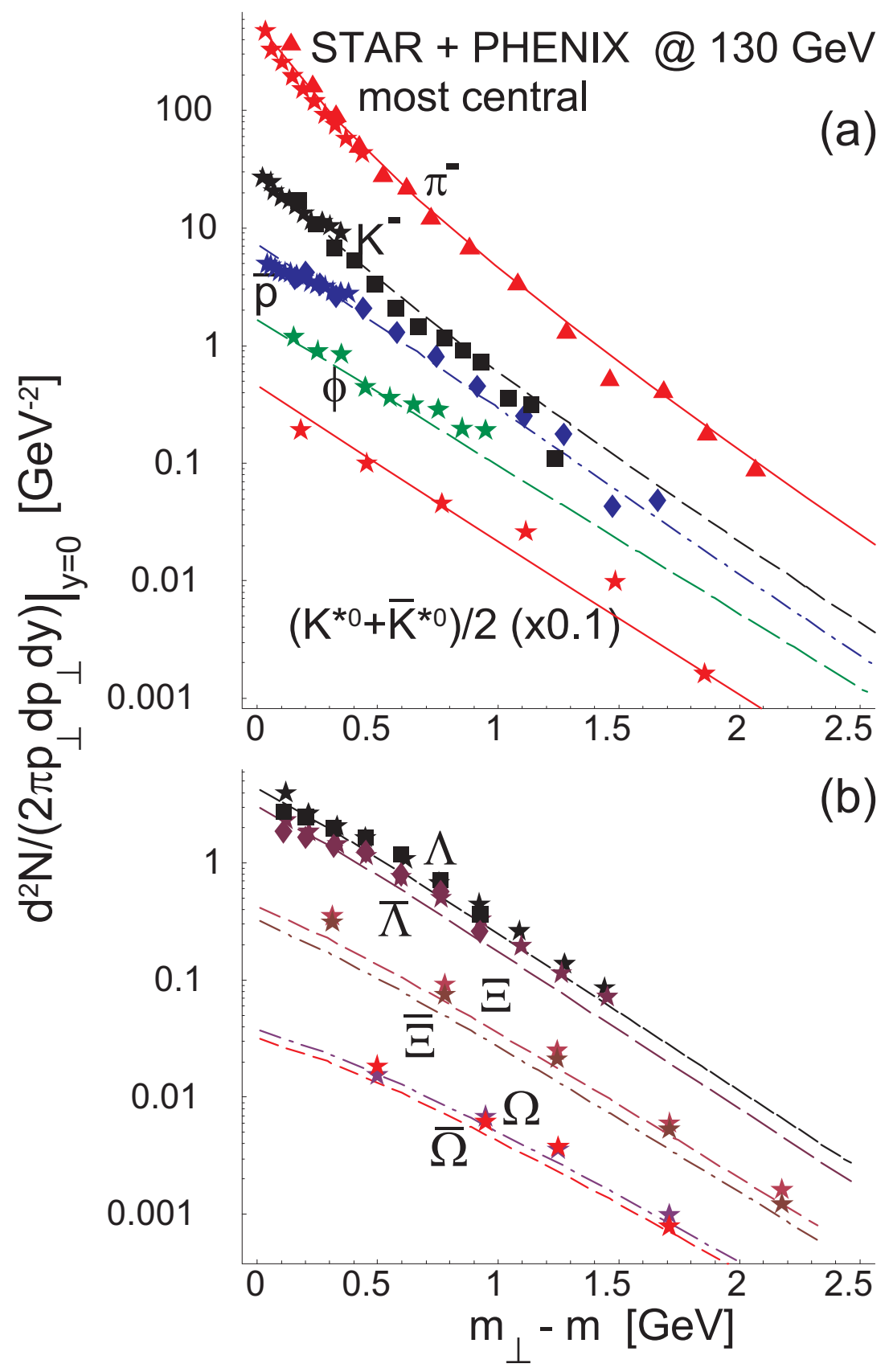

FIGURE 1. The transverse-mass spectra at midrapidity. The data from STAR are denoted by asterisks, other symbols are used to denote the PHENIX data. All spectra are for most central collisions

spectra of pions, kaons and protons, which gave us, for the most central events, the following values of the size parameters:

$$
\tau=7.66 \mathrm{fm}, \quad \rho_{\max }=6.69 \mathrm{fm} .
$$


In Fig. 1 we show our results for all up-to-now available spectra at $\sqrt{s_{N N}}=130 \mathrm{GeV}$ for the most-central collisions. In the upper part of Fig. 1 we show the spectra of pions, kaons, and antiprotons (used earlier to determine the geometric parameters) and the predicted spectra of the $\phi$ and $K^{*}(892)^{0}$ mesons. The predicted spectrum of the $\phi$ mesons agrees very well with the measurement [41], with model curve crossing five out of the nine data points. The $\phi$ meson production is of a particular interest in relativistic heavy-ion collisions, since its spectrum reflects the initial temperature of the hadronic system. This is because its interaction with the hadronic environment is negligible. Moreover, it does not receive any contribution from resonance decays. Thus, the agreement of the model and the data supports the idea of one universal freeze-out. The upper part of Fig. 1 also shows the averaged spectrum of $K^{*}$ resonances, with the data from Ref. [13]. Once again we observe a very good agreement between the model curve and the experimental points. As already mentioned above, the successful description of both the yield and the spectrum of $K^{*}(892)^{0}$ mesons supports the concept of the thermal description of hadron production at RHIC, and brings evidence for a very small interval between chemical and thermal freeze-outs.

In the lower part of Fig. 1 we show the predictions of the model for the spectra of hyperons. Again, in view of the fact that no extra parameters are introduced here and no refitting has been performed, the agreement is impressive. We note that the preliminary [46] data for the $\Xi$ 's used in the figure were subsequently updated, which resulted in the reduction of the data by about a factor of 2 . This correction makes our agreement with the data even better. The data accumulated at lower energies at SPS showed that the slope of the $\Omega$ hyperon was much steeper than for other particles [47]. On the contrary, in the case of RHIC the model predictions for the $\Omega$ are as good as for the other hadrons. Since the $\Omega$ contains three strange quarks, it is most sensitive for modifications of the simple thermal model used here, e.g. the use of canonical instead of the grand-canonical ensemble. The agreement of Fig. 1 does not support the need for inclusion of these effects.

\section{SUMMARY}

The success of our model in reproducing the hadron ratios and the transverse-mass spectra indicates that the particles are indeed produced thermally. The model has only four parameters and describes the data with surprising accuracy. Since our approach uses hadronic degrees of freedom and starts at freeze-out, important theoretical questions concerning the earlier stages of the evolution cannot be addressed in this framework. We think, however, that our results constrain any more microscopic descriptions of the evolution of the matter formed in ultra-relativistic heavy-ion collisions. Further applications of the model aiming at the description of the elliptic flow and the HBT pion radii will be presented in the next contribution to these Proceedings [10]. 


\section{ACKNOWLEDGMENTS}

Supported in part by the Polish State Committee for Scientific Research, grant 2 P03B 09419. WB acknowledges the support of PRAXIS XXI/BCC/429/94.

\section{REFERENCES}

1. Broniowski, W., and Florkowski, W., Phys. Rev. Lett., 87, 272302 (2001).

2. Broniowski, W., and Florkowski, W., Phys. Rev. C, 65, 064905 (2002).

3. Broniowski, W., Baran, A., and Florkowski, W., Acta Phys. Pol. B, 33, 4235 (2002).

4. Florkowski, W., Broniowski, W., and Michalec, M., Acta Phys. Pol. B, 33, 761 (2002).

5. Michalec, M., Thermal description of particle production in ultra-relativistic heavy-ion collisions, Ph.D. thesis, Institute of Nuclear Physics, ul. Radzikowskiego 152, 31-342 Kraków, Poland (2001), available as nucl-th/0112044.

6. Broniowski, W., and Florkowski, W., Acta Phys. Pol. B, 33, 1935 (2002).

7. Broniowski, W., and Florkowski, W., "Thermal model at RHIC: particle ratios and $p_{\perp}$ spectra," in Ultrarelativistic Heavy-Ion Collisions, edited by M. Buballa, W. Nörenberg, B.-J. Schaefer, and J. Wambach (GSI, Darmstadt) Hirschegg, Austria, 2002, p. 146, hep-ph/0202059.

8. Florkowski, W., and Broniowski, W., Acta Phys. Pol. B, 33, 1629 (2002).

9. Florkowski, W., and Broniowski, W., "Thermal description of transverse-momentum spectra at RHIC," in Proceedings of Quark Matter 2002 Conference, Nucl. Phys. A in print, nucl-th/0208061.

10. Broniowski, W., Baran, A., and Florkowski, W., "Thermal model at RHIC, part II: elliptic flow and HBT radii," following paper.

11. Bjorken, J. D., Phys. Rev. D, 27, 140 (1983).

12. Heinz, U., Nucl. Phys. A, 661, 140c (1999).

13. Xu, Z., STAR Collaboration, Nucl. Phys. A, 698, 607c (2002).

14. Braun-Munzinger, P., Magestro, D., Redlich, K., and Stachel, J., Phys. Lett. B, 518, 41 (2001).

15. Rafelski, J., and Letessier, J., Phys. Rev. Lett., 85, 4695 (2000).

16. Hagedorn, R., Suppl. Nuovo Cim., 3, 147 (1965).

17. Broniowski, W., and Florkowski, W., Phys. Lett. B, 490, 223 (2000).

18. Broniowski, W., "Distinct Hagedorn temperatures from the particle spectra: a higher one for mesons, a lower one for baryons," in Few-Quark Problems, edited by B. Golli, M. Rosina, and S. Širca, Bled, Slovenia, 2002, p. 14, hep-ph/0008112.

19. Tounsi, A., Letessier, J., and Rafelski, J., "Hadronic matter equation of state and the hadron mass spectrum," in Hot Hadronic Matter, Divonne-les-Bains, France, 1994, p. 105.

20. Braun-Munzinger, P., Heppe, I., and Stachel, J., Phys. Lett. B, 465, 15 (1999).

21. Gaździcki, M., and Gorenstein, M. I., Acta Phys. Pol. B, 30, 2705 (1999).

22. Yen, G. D., and Gorenstein, M. I., Phys. Rev. C, 59, 2788 (1999).

23. Becattini, F., Cleymans, J., Keranen, A., Suhonen, E., and Redlich, K., Phys. Rev. C, 64, 024901 (2001).

24. Baym, G., Friman, B., Blaizot, J. P., Soyeur, M., and Czyż, W., Nucl. Phys. A, 407, 541 (1983).

25. Milyutin, P., and Nikolaev, N. N., Heavy Ion Phys., 8, 333 (1998).

26. Siemens, P. J., and Rasmussen, J., Phys. Rev. Lett., 42, 880 (1979).

27. Schnedermann, E., Sollfrank, J., and Heinz, U., Phys. Rev. C, 48, 2462 (1993).

28. Csörgö, T., and Lörstad, B., Phys. Rev. C, 54, 1390 (1996).

29. Rischke, D. H., and Gyulassy, M., Nucl. Phys. A, 597, 701 (1996).

30. Scheibl, R., and Heinz, U., Phys. Rev. C, 59, 1585 (1999).

31. Cooper, F., and Frye, G., Phys. Rev. D, 10, 186 (1974).

32. Cooper, F., Frye, G., and Schonberg, E., Phys. Rev. D, 11, 192 (1975).

33. Karsch, F., Nucl. Phys. A, 698, 199c (2002).

34. Becattini, F., J. Phys. G, 28, 1553 (2002).

35. Back, B. B., et al., PHOBOS Collaboration, Phys. Rev. Lett., 87, 102301 (2001).

36. Bearden, I. G., BRAHMS Collaboration, Nucl. Phys. A, 698, 667c (2002). 
37. Harris, J., STAR Collaboration, Nucl. Phys. A, 698, 64c (2002).

38. Adams, J., et al., STAR Collaboration, nucl-ex/0211024.

39. Ohnishi, H., PHENIX Collaboration, Nucl. Phys. A, 698, 659c (2002).

40. Caines, H., STAR Collaboration, Nucl. Phys. A, 698, 112c (2002).

41. Adler, C., et al., STAR Collaboration, Phys. Rev. C, 65, 041901 (2002).

42. Adler, C., et al., STAR Collaboration, Phys. Rev. Lett., 89, 092301 (2002).

43. Adler, C., et al., STAR Collaboration, Phys. Rev. Lett., 87, 262302 (2001).

44. Suire, C., STAR Collaboration, nucl-ex/0211017.

45. Castillo, J., STAR Collaboration, nucl-ex/0210032.

46. Castillo, J., STAR Collaboration, J. Phys. G., 28, 1987 (2002).

47. Antinori, C., et al., WA97 Collaboration, J. Phys. G., 27, 375 (2001). 